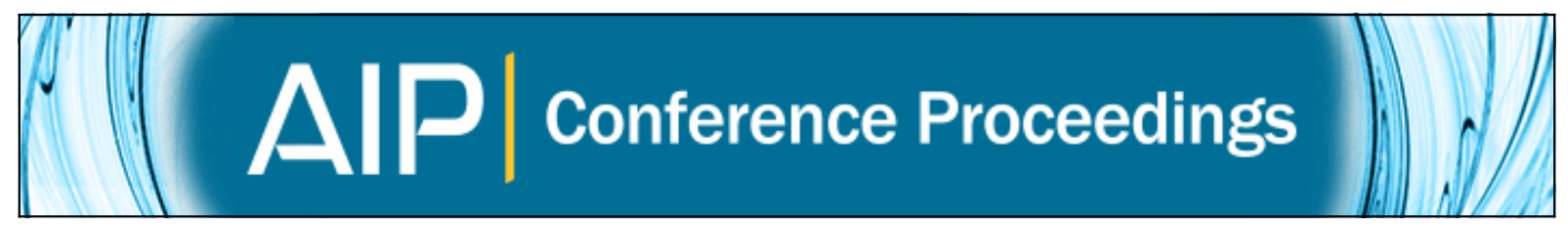

\title{
Efficiency and stability of the DSBGK method
}

Jun Li

Citation: AIP Conference Proceedings 1501, 849 (2012); doi: 10.1063/1.4769631

View online: http://dx.doi.org/10.1063/1.4769631

View Table of Contents: http://scitation.aip.org/content/aip/proceeding/aipcp/1501?ver=pdfcov

Published by the AIP Publishing

Articles you may be interested in

Open-loop control of noise amplification in a separated boundary layer flow

Phys. Fluids 25, 124106 (2013); 10.1063/1.4846916

A modulated gradient model for scalar transport in large-eddy simulation of the atmospheric boundary layer Phys. Fluids 25, 015110 (2013); 10.1063/1.4774342

Molecular simulation of small Knudsen number flows

AIP Conf. Proc. 1501, 864 (2012); 10.1063/1.4769633

Investigation of rarefied supersonic flows into rectangular nanochannels using a three-dimensional direct simulation Monte Carlo method

Phys. Fluids 22, 032001 (2010); 10.1063/1.3302805

Molecular transition and slip flows in the pumping channels of drag pumps

J. Vac. Sci. Technol. A 18, 1025 (2000); 10.1116/1.582294 


\title{
Efficiency and Stability of the DSBGK Method
}

\author{
Jun Li \\ Mathematical and Computer Sciences \& Engineering, KAUST, Thuwal 23955-6900, Saudi Arabia
}

\begin{abstract}
Recently, the DSBGK method (Note: the original name DS-BGK is changed to DSBGK for simplicity) was proposed to reduce the stochastic noise in simulating rarefied gas flows at low velocity. Its total computational time is almost independent of the magnitude of deviation from equilibrium state. It was verified by the DSMC method in different benchmark problems over a wide range of $K n$ number. Some simulation results of the closed lid-driven cavity flow, thermal transpiration flow and the open channel flow by the DSBGK method are given here to show its efficiency and numerical stability. In closed problems, the density distribution is subject to unphysical fluctuation due to the absence of density constraint at the boundary. Thus, many simulated molecules are employed by DSBGK simulations to improve the stability and reduce the magnitude of fluctuation. This increases the memory usage remarkably but has small influence to the efficiency of DSBGK simulations. In open problems, the DSBGK simulation remains stable when using about 10 simulated molecules per cell because the fixed number densities at open boundaries eliminate the unphysical fluctuation. Small modification to the CLL reflection model is introduced to further improve the efficiency slightly.
\end{abstract}

Keywords: micro gas flows, variance reduction, BGK equation, DSMC method, surface reflection.

PACS: 45.20.Jj, 42.25.Gy, 34.10.+x, 02.60.Cb.

\section{INTRODUCTION}

For micro gas flows, the Boltzmann equation rather than the Navier-Stokes equation should be used due to high $K n$ number. In addition, the influence of boundary condition to the solutions becomes dominant because the frequency of molecular reflection on the solid wall, compared to the frequency of intermolecular collisions, increases with $K n$. Unfortunately, the characteristic velocity of micro gas flows is usually much smaller than the molecular random thermal velocity and sometimes the variations of quantities of interest inside the flow domain are very small, which makes it time-consuming to get smooth results by the traditional particle simulation methods.

The DSBGK method was proposed in [1] to improve the efficiency in simulating micro gas flows and verified in the lid-driven, Couette and channel flow problems [1-3] by comparison with the DSMC method [4] as criterion. Few tentative results of the DSBGK simulations using the CLL reflection model $[5,6]$ were verified by the DSMC results in [2]. Although based on the BGK equation obtained by using a simple model to replace the intermolecular collision integral of the Boltzmann equation, the DSBGK method agrees well with the DSMC method at $K n=0.063$ and 6.3 in the lid-driven cavity flow [1]. This is because the molecular reflection on the wall, the dominant effect in micro gas flows, is modeled by the DSBGK method in the same way as by the DSMC method. Theoretically, the error due to simplification to the intermolecular collision process vanishes and the solution depends only on the boundary condition when $K n \rightarrow \infty$. The DSBGK method achieves high efficiency by avoiding generating random fractions in the intermolecular collision process and using the increments (instead of transient values) of molecular variables to update cell's macro quantities, which significantly reduces the statistical noise due to discontinuous events of simulated molecules moving into and out of cells. Theoretical analysis to the algorithms of DSMC and DSBGK methods and the detailed comparison of simulation results between the two methods were given in [3].

The benchmark problems are divided into closed and open problems to discuss the efficiency and stability of the DSBGK simulation separately. In closed problems, the long-period fluctuation is observed in the number density distribution of DSBGK simulations. Many simulated molecules are employed to reduce the magnitude of fluctuation and to improve the numerical stability. Consequently, the memory usage is increased remarkably but the efficiency is still very high as shown in the closed lid-driven problem [1]. In open problems, the boundary condition with fixed number density eliminates the unphysical fluctuation and the DSBGK simulation remains stable even when using about 10 simulated molecules per cell, which significantly reduces the memory usage and improves the applicability in open problems of large scale. The selection of relaxation parameter of the BGK equation is discussed. 


\section{ALGORITHM OF THE DSBGK METHOD}

In the DSBGK simulation [1,3], each simulated molecule carries: position $\mathbf{x}_{l}$, velocity $\mathbf{c}_{l}$, number $N_{l}$ of real molecules represented by the simulated molecule $l$, and $F_{l}$ which is the representative value $f\left(t, \mathbf{x}_{l}, \mathbf{c}_{l}\right)$ of $f$ at the moment $t$ and the point $\left(\mathbf{x}_{l}, \mathbf{c}_{l}\right)$. During the simulation process, the representative sample $\left[\mathbf{x}_{l}, \mathbf{c}_{l}, N_{l}\right]_{\text {all }}$ of $f$ is updated to represent the evolution of $f$ due to molecular motions and intermolecular collisions by deterministically changing $\mathbf{x}_{l}$ and $N_{l}$ rather than $\mathbf{c}_{l}$. Meanwhile, $F_{l}$ is updated with $\mathbf{x}_{l}$ and used to update $N_{l}$ by the extrapolation [1] of the acceptance-rejection method. In the molecular reflection process at $\mathbf{x}_{l}$ on the wall, $N_{l}$ remains unchanged to conserve mass and $\mathbf{c}_{l}$ is changed randomly to $\mathbf{c}_{l, \text { new }}$ to represent the evolution of $f$. Consequently, $F_{l}$ is updated to $f\left(t, \mathbf{x}_{l}, \mathbf{c}_{l, \text { new }}\right)$ where $\mathbf{c}_{l, \text { new }}=\mathbf{c}_{\mathrm{r}}+\mathbf{u}_{\text {wall }}, \mathbf{c}_{\mathrm{r}}$ is selected randomly and $\mathbf{u}_{\text {wall }}$ is the wall velocity. It is easy to prove that the compatibility condition, namely $\left[\mathbf{x}_{l}, \mathbf{c}_{l}, N_{l}\right]_{\text {all }}$ and $\left[F_{l}\right]_{\text {all }}$ are related to the same $f$, is satisfied during the simulation process. In addition, three transitional cell's quantities, namely the number density $n_{\mathrm{tr}, k}$, flow velocity $\mathbf{u}_{\mathrm{tr}, k}$ and temperature $T_{\mathrm{tr}, k}$, are used to construct a transitional Maxwell distribution function $f_{\text {eq,tr, }, k}$, which is used in each cell $k$ to replace the original $f_{\text {eq }}$ defined by the unknown $n, \mathbf{u}, T$. The DSBGK algorithm is summarized as follows:

At the initial state, $n_{\mathrm{tr}, k}, \mathbf{u}_{\mathrm{tr}, k}, T_{\mathrm{tr}, k}$ are equal to the initial macro quantities which are usually uniform. $\mathbf{x}_{l}$ and $\mathbf{c}_{l}$ are selected randomly as in the DSMC simulation and then $F_{l}$ is equal to $f_{\text {eq, tr }, k}\left(0, \mathbf{x}_{l}, \mathbf{c}_{l}\right)$. The initial values $N_{l, t=0}$ of $N_{l}$ for different simulated molecules are usually the same and selected appropriately such that the total number of simulated molecules, namely $N_{\text {total,real }} / N_{l, t=0}$ where $N_{\text {total,real }}$ is the total number of real molecules, takes a acceptable value.

During each $\Delta t$, each simulated molecule moves uniformly and in a straight line before encountering the boundary. The molecular trajectory during each $\Delta t$ may be divided into several segments by cell's interfaces or remains as a single segment if not yet arriving at any cell's interface at the end of current $\Delta t$. As each segment is located inside a particular cell $k, F_{l}$ is conveniently updated along each segment in sequence according to the Lagrangian form of the BGK equation using $f_{\text {eq,tr }, k}$ which is constant for each segment. Meanwhile, $N_{l}$ is updated to make the ratio $N_{l} / F_{l}$ constant which is based on the extrapolation of acceptance-rejection scheme (Note: $F_{l}$ is changed with $\mathbf{c}_{l}$ but $N_{l}$ remains unchanged during the molecular reflection process on the wall). At the end of each $\Delta t$, the increments of $N_{l}$ of all related simulated molecules due to intermolecular collisions inside the same cell $k$ during the same $\Delta t$ are used to update $n_{\mathrm{tr}, k}, \mathbf{u}_{\mathrm{tr}, k}, T_{\mathrm{tr}, k}$ according to auto-regulation schemes based on the mass, momentum and energy conservation principles, which make $n_{\mathrm{tr}, k}, \mathbf{u}_{\mathrm{tr}, k}, T_{\mathrm{tr}, k}$ converging to the solutions of $n, \mathbf{u}, T$ of the BGK equation $[1,3]$.

For open problems, simulated molecules are removed from the flow domain when moving across the open boundary during each $\Delta t$. Correspondingly, some new simulated molecules are generated at the end of each $\Delta t$ at the open boundary with $\mathbf{x}_{l}$ and $\mathbf{c}_{l}$ being selected randomly as in the DSMC simulation. Then, $F_{l}$ is determined from $\mathbf{x}_{l}$ and $\mathbf{c}_{l}$ through $f_{\text {eq,tr, } k}$ using the macro quantities fixed at the open boundary or the values of adjacent cell if not prescribed at the boundary. The initial values of $N_{l}$ of new simulated molecules at different parts of the open boundary can be different in DSBGK simulations. In the channel flow problem driven by the density difference $\Delta n_{\text {end }}$ at the two ends [2,3], we use different initial values $N_{l \text {,init,end }}$ for different ends such that their ratios of $N_{l \text {,init,end }} / n_{\text {end }}$ are equal, which makes the number of simulated (not real) molecules per cell almost the same for different cells having the same volume but different number density of real molecules. As the stochastic noise at each cell depends on the average number of simulated molecules inside that cell, such selection of the initial values of $N_{l}$ for new simulated molecules at different parts of the open boundary achieves the trade-off of stochastic noise among cells and so reduces the sample size required for getting smooth results in the whole flow domain.

When molecule $l$ reflecting at $\mathbf{x}_{l}$ on the wall, $\mathbf{c}_{l}$ is changed to $\mathbf{c}_{l, \text { new }}=\mathbf{c}_{\mathbf{r}}+\mathbf{u}_{\text {wall }}$ where $\mathbf{c}_{\mathrm{r}}$ selected randomly according to the reflection model as in the DSMC simulation. Then, $F_{l}$ is updated to $f\left(t, \mathbf{x}_{l}, \mathbf{c}_{l, \text { new }}\right)$. The subscript $l$ is omitted in the expression of component. We predetermine a local Cartesian reference system $S_{\text {local }}$ moving at $\mathbf{u}_{\text {wall }}$, in which the subscripts 2 and 3 represent the tangential directions of wall. The incoming velocity is $\mathbf{c}_{\mathrm{i}}=\mathbf{c}_{l}-\mathbf{u}_{\mathrm{wall}}$. As $\mathbf{c}_{l}$ and $\mathbf{u}_{\mathrm{wall}}$ are stored in the component form of the unique global Cartesian reference system $\mathrm{S}_{\text {global }}$, we need the transformation from $\mathrm{S}_{\text {global }}$ to $\mathrm{S}_{\text {local }}$ to obtain the components $c_{\mathrm{i}, 1}, c_{\mathrm{i}, 2}, c_{\mathrm{i}, 3}$. Finally, $c_{\mathrm{r}, 1}, c_{\mathrm{r}, 2}, c_{\mathrm{r}, 3}$ are transformed from $\mathrm{S}_{\text {local }}$ to $\mathrm{S}_{\mathrm{global}}$ to obtain $\mathbf{c}_{l, \text { new }}$ in $\mathrm{S}_{\text {global. }}$. For selecting the unit normal vector $\mathbf{e}_{\mathrm{n}}$, we specify that $c_{\mathrm{i}, 1}$ is negative and $c_{\mathrm{r}, 1}=\mathbf{c}_{\mathrm{r}} \cdot \mathbf{e}_{\mathrm{n}}$ is positive. In the reflection process, the subscripts $1,2,3$ always represent the related components in $\mathrm{S}_{\text {local }}$. In the original CLL model $[5,6]$, we compute the tangential components by $c_{\mathrm{r}, 2}=v \cos \theta-w \sin \theta$ and $c_{\mathrm{r}, 3}=v \sin \theta+w \cos \theta$ where $\nu=\left[\left(1-\alpha_{\tau}\right)\left(c_{\mathrm{i}, 2} c_{\mathrm{i}, 2}+c_{\mathrm{i}, 3} c_{\mathrm{i}, 3}\right)\right]^{1 / 2}+\left(2 k_{\mathrm{B}} T_{\mathrm{wall}} / m\right)^{1 / 2} r_{\tau} \cos \varphi_{\tau}, \quad w=\left(2 k_{\mathrm{B}} T_{\mathrm{wall}} / m\right)^{1 / 2} r_{\tau} \sin \varphi_{\tau}, \quad r_{\tau}=\left(-\alpha_{\tau} \ln R f_{1}\right)^{1 / 2}, \quad \varphi_{\tau}=2 \pi R f_{2}, \quad k_{\mathrm{B}} \quad$ is the Boltzmann constant, $m$ is the molecular mass, $\theta$ is the azimuthal angle of incoming velocity components $\left(c_{\mathrm{i}, 2}, c_{\mathrm{i}, 3}\right)$ in the $x_{2} x_{3}$ plane of $\mathrm{S}_{\text {local }}, R f_{1}$ and $R f_{2}$ are two different random fractions distributed uniformly inside [0, 1], $\alpha_{\tau}$ is the accommodation coefficient of kinetic energy of the tangential velocity component. For the normal component, $c_{\mathrm{r}, 1}=\left[\left(1-\alpha_{\mathrm{n}}\right) c_{\mathrm{i}, 1} c_{\mathrm{i}, 1}+\left(2 k_{\mathrm{B}} T_{\mathrm{wall}} / m\right) r_{\mathrm{n}} r_{\mathrm{n}}+2\left(1-\alpha_{\mathrm{n}}\right)^{1 / 2}\left|c_{\mathrm{i}, 1}\right|\left(2 k_{\mathrm{B}} T_{\mathrm{wall}} / m\right)^{1 / 2} r_{\mathrm{n}} \cos \varphi_{\mathrm{n}}\right]^{1 / 2}$ where $\left|c_{\mathrm{i}, 1}\right|$ is the absolute value of $c_{\mathrm{i}, 1}$, $r_{\mathrm{n}}=\left(-\alpha_{\mathrm{n}} \ln R f_{3}\right)^{1 / 2}, \varphi_{\mathrm{n}}=2 \pi R f_{4}, R f_{3}$ and $R f_{4}$ are two additional random fractions and $\alpha_{\mathrm{n}}$ is the accommodation coefficient 
of kinetic energy of the normal velocity component. We get $c_{\mathrm{r}, 2}=c_{\mathrm{i}, 2}\left(1-\alpha_{\tau}\right)^{1 / 2}+\left(2 k_{\mathrm{B}} T_{\mathrm{wall}} / m\right)^{1 / 2} r_{\tau} \cos \left(\varphi_{\tau}+\theta\right)$ and $c_{\mathrm{r}, 3}=c_{\mathrm{i}, 3}\left(1-\alpha_{\tau}\right)^{1 / 2}+\left(2 k_{\mathrm{B}} T_{\text {wall }} / m\right)^{1 / 2} r_{\tau} \sin \left(\varphi_{\tau}+\theta\right)$ after reorganizing the above formulas. Note that $\varphi_{\tau}$ is selected uniformly from a periodic interval $[0,2 \pi]$ and so $\varphi_{\tau}+\theta$ can be replaced simply by $\varphi_{\tau}$, which implies that the calculation of $\theta$ can be avoided to slightly improve the efficiency. So, the equivalent but simpler algorithm to compute the tangential components in $\mathrm{S}_{\text {local }}$ is that $c_{\mathrm{r}, 2}=c_{\mathrm{i}, 2}\left(1-\alpha_{\tau}\right)^{1 / 2}+\left(2 k_{\mathrm{B}} T_{\mathrm{wall}} / m\right)^{1 / 2} r_{\tau} \cos \varphi_{\tau}$ and $c_{\mathrm{r}, 3}=c_{\mathrm{i}, 3}\left(1-\alpha_{\tau}\right)^{1 / 2}+\left(2 k_{\mathrm{B}} T_{\mathrm{wall}} / m\right)^{1 / 2} r_{\tau} \sin \varphi_{\tau}$ [2]. This simpler algorithm for the CLL model also degenerates to the Maxwell diffuse reflection model when $\alpha_{\tau}=\alpha_{\mathrm{n}}=1$.

Now, $F_{l}$ should be updated to $F_{l, \text { new }}=f\left(t, \mathbf{x}_{l}, \mathbf{c}_{l, \text { new }}\right)=f\left(t, \mathbf{x}_{l}, \mathbf{c}_{\mathrm{r}}+\mathbf{u}_{\text {wall }}\right)$. Note that $F_{l}$ is the representative value of volume distribution $f$ which is different from the scattering kernel $R$ used to select $\mathbf{c}_{\mathrm{r}}$ for each particular reflection process. We introduce $f_{\mathrm{B}}(\mathbf{c})$ as the observed distribution function of $f$ in $\mathrm{S}_{\text {local }}$ at the reflection point $\mathbf{x}_{l}$ and at the current moment $t$, which means $f_{\mathrm{B}}(\mathbf{c})=f\left(t, \mathbf{x}_{l}, \mathbf{c}+\mathbf{u}_{\text {wall }}\right)$. After getting the formula of $f_{\mathrm{B}}(\mathbf{c}), F_{l, \text { new }}=f_{\mathrm{B}}\left(\mathbf{c}_{\mathrm{r}}\right)$. The distribution $f_{\mathrm{B}}\left(\mathbf{c}_{\mathrm{i}}\right)$ of the incoming molecules is known from the molecular information in the adjacent cell. $f_{\mathrm{B}}\left(\mathbf{c}_{\mathrm{r}}\right)$ is the distribution of reflecting molecules and determined from the scattering kernel and the incoming mass flux. For the Maxwell diffuse reflection model, we have $f_{\mathrm{B} \text {,diffuse }}\left(\mathbf{c}_{\mathrm{r}}\right)=n_{\mathrm{efff}}\left[m /\left(2 \pi k_{\mathrm{B}} T_{\text {wall }}\right)\right]^{3 / 2} \exp \left[-m\left|\mathbf{c}_{\mathrm{r}}\right|^{2} /\left(2 k_{\mathrm{B}} T_{\text {wall }}\right)\right]$ where the effective number density is $n_{\mathrm{eff}}=n_{\mathrm{tr}, k}\left(T_{\mathrm{tr}, k} / T_{\text {wall }}\right)^{1 / 2}\left\{\exp \left[-\left(u_{\text {in }}^{\prime}\right)^{2}\right]+\pi^{1 / 2} u_{\text {in }}^{\prime}\left[1+\operatorname{erf}\left(u_{\text {in }}^{\prime}\right)\right]\right\}, u_{\text {in }}^{\prime}=\left[-\left(\mathbf{u}_{\mathrm{tr}, k}-\mathbf{u}_{\mathrm{wall}}\right) \cdot \mathbf{e}_{\mathrm{n}}\right] \times\left[m /\left(2 k_{\mathrm{B}} T_{\mathrm{tr}, k}\right)\right]^{1 / 2}$ is the normalized incoming component of $\mathbf{u}_{\mathrm{tr}, k}$ relative to the moving wall. $n_{\mathrm{tr}, k}, \mathbf{u}_{\mathrm{tr}, k}$ and $T_{\mathrm{tr}, k}$ are the values of the cell $k$ adjacent to the reflection point $\mathbf{x}_{l}$. We store $n_{\text {eff }}$ and use it repeatedly for different simulated molecules reflecting on the same subarea close to cell $k$ during the same $\Delta t$ and update $n_{\text {eff }}$ at the end of each $\Delta t$. The tentative formula of $f_{\mathrm{B}}\left(\mathbf{c}_{\mathrm{r}}\right)$ for the CLL reflection model is given in [1] and discussed in detail in [3].

When considering external body force, we split $\partial f / \partial t$ into $\partial f /\left.\partial t\right|_{\text {move }}=-\mathbf{c} \cdot \partial f / \partial \mathbf{x}, \partial f /\left.\partial t\right|_{\text {coll }}=v\left(f_{\text {eq }}-f\right)$ and $\partial f /\left.\partial t\right|_{\text {force }}=-\mathbf{a} \cdot \partial f / \partial \mathbf{c}$ where $\mathbf{a}$ is the acceleration due to external force. To simplify the algorithm, we decouple the effect due to $\partial f /\left.\partial t\right|_{\text {force }}$ from the other two effects. At the end of each $\Delta t$ of the original DSBGK algorithm, the effects due to $\partial f /\left.\partial t\right|_{\text {move }}$ and $\partial f /\left.\partial t\right|_{\text {coll }}$ are already incorporated into the simulation and so we consider $\partial f /\left.\partial t\right|_{\text {force }}$ by changing $\mathbf{c}_{l}$ of each simulated molecule to $\mathbf{c}_{l}+\Delta t \mathbf{a}$ with $\mathbf{x}_{l}, F_{l}, N_{l}$ keeping unchanged as $f(t+\Delta t, \mathbf{x}+\Delta t \mathbf{c}, \mathbf{c}+\Delta t \mathbf{a})=f(t, \mathbf{x}, \mathbf{c})$ if neglecting intermolecular collision. Correspondingly, $\mathbf{u}_{\mathrm{tr}, k}$ of each cell is changed to $\mathbf{u}_{\mathrm{tr}, k}+\Delta t \mathbf{a}$ with $n_{\mathrm{tr}, k}, T_{\mathrm{tr}, k}$ keeping unchanged. When sampling and outputting the cell's velocity, we use the average value before and after implementing $\mathbf{a}$, namely $\mathbf{u}_{\mathrm{tr}, k}+0.5 \Delta t \mathbf{a}$.

In DSBGK simulation, we use $v=v(\mu) \equiv n k_{\mathrm{B}} T / \mu$ for problems where the momentum exchange is the dominant effect and use $v=v(\kappa) \equiv 5 n k_{\mathrm{B}}^{2} T /(2 m \kappa)$ if the heat conduction is the dominant effect. For monoatomic gas where $P r=2 / 3$ and $C_{p}=5 k_{\mathrm{B}} /(2 m)$, we compute $v(\kappa)$ by $v(\kappa)=2 n k_{\mathrm{B}} T /(3 \mu)$ as $\kappa=C_{p} \mu / \operatorname{Pr}$ and $\mu$ is given usually.

\section{SIMULATION RESULTS BY THE DSBGK METHOD}

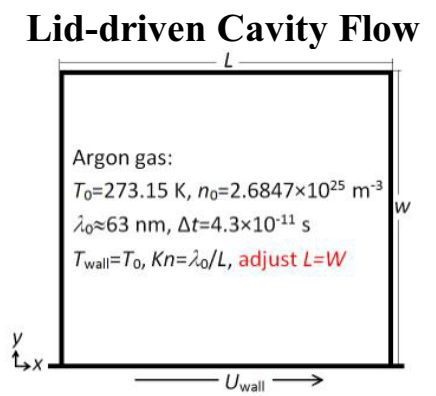

FIGURE 1. Schematic model of closed lid-driven cavity flow.

The DSBGK simulations of this problem were briefly reported in [1]. The Maxwell boundary condition is used and cell number is $20 \times 20$ for $K n=0.063$ and 6.3. Each cell contains about 2000 simulated molecules and $v=v(\mu)$ in the DSBGK simulations. To show the high efficiency of DSBGK simulation at low velocity, we set $U_{\text {wall }}=0.1 \mathrm{~m} / \mathrm{s}$. Fig. 2 shows the transient DSBGK results (no average) at $600^{\text {th }} \Delta t$ taking about 7 minute of computational time of one CPU on Lenovo laptop E43A. We can output many transient results at different moments of interest at the cost of very small increase of CPU time used for writing data to the hard disc. From the efficiency point of view, the DSBGK method is a promising tool for studying transient problem. But, the hysteresis effect of DSBGK simulations, which are based on auto-regulation scheme, was observed in [2]. New techniques, like time rescaling, are required to reduce the magnitude of hysteresis effect. We increase $U_{\text {wall }}$ to $20 \mathrm{~m} / \mathrm{s}$ and $K n$ to 6.3 . The DSBGK transient results at $40^{\text {th }} \Delta t$ agree very well with the DSMC results. The DSBGK distributions of $T, u, v$ remain unchanged after $40 \Delta t$. But, the DSBGK distribution of $n$ cannot stay at steady state and its deviation at $900^{\text {th }} \Delta t$ from the DSMC results is remarkable. This drawback of the DSBGK method in closed problems implies that the ensemble-average process (if 
necessary) should be used for quantities related to $n$. In open problems, the unphysical fluctuation of $n$ is eliminated by the fixed $n$ at open boundaries and so the more-efficient time-average process can be used (see the following results of channel flow). The DSBGK simulation at $K n=6.3$ within $40 \Delta t$ takes about 11 minutes of CPU time.

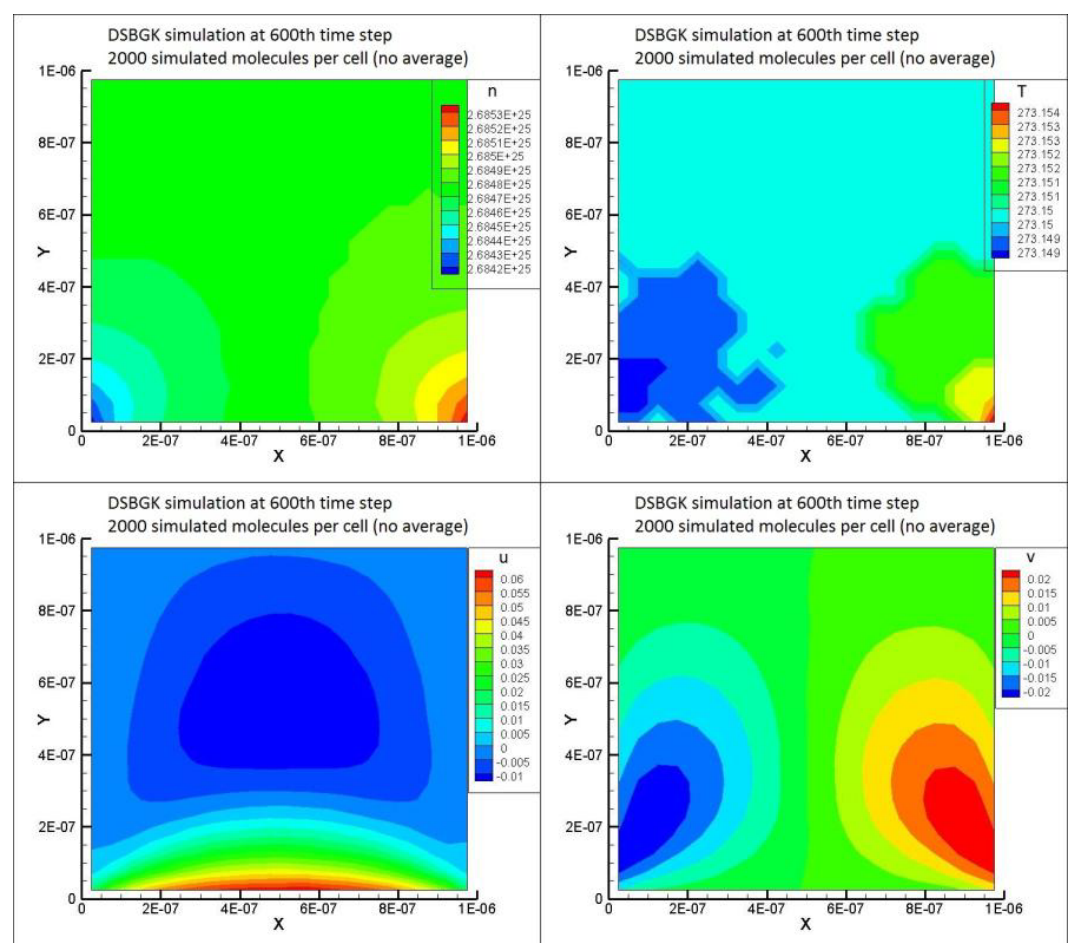

FIGURE 2. Transient DSBGK results at $600^{\text {th }} \Delta t, K n=0.063, U_{\text {wall }}=0.1 \mathrm{~m} / \mathrm{s}, 7$ minutes of CPU time.

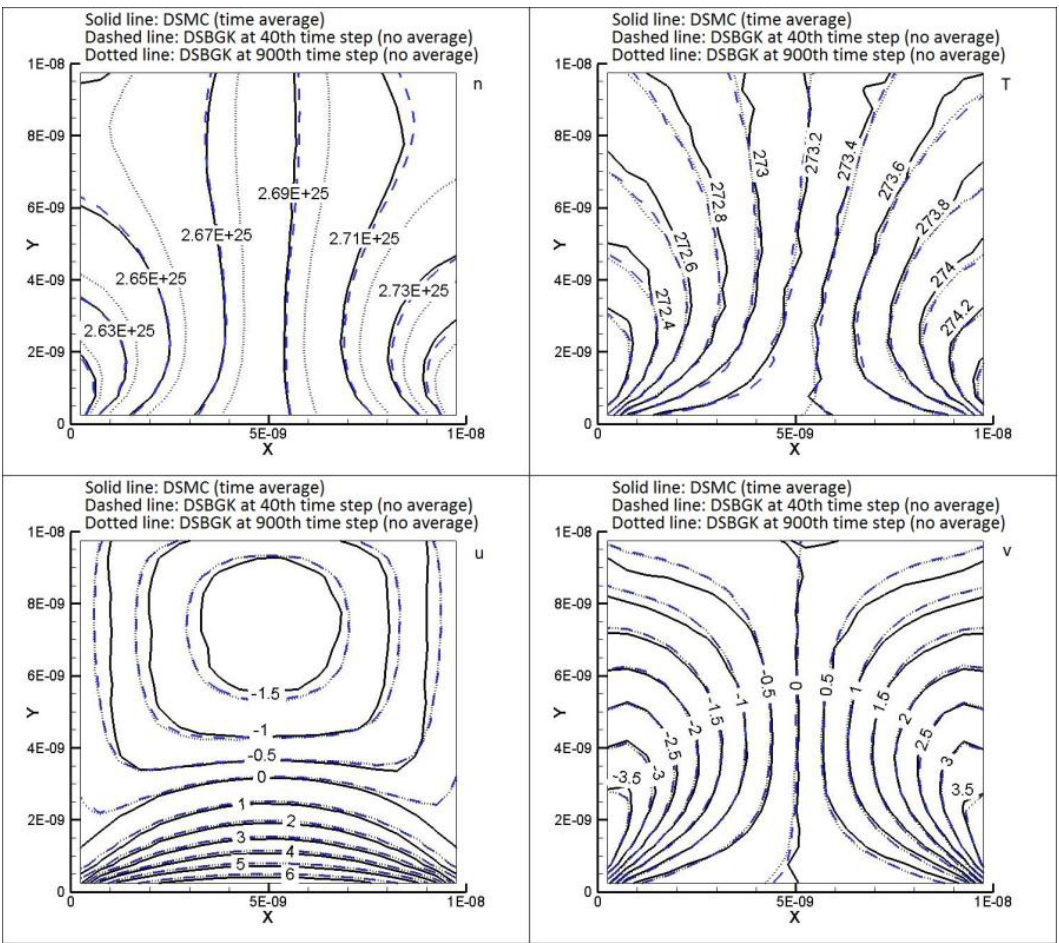

FIGURE 3. Comparison between DSMC and DSBGK methods, $K n=6.3, U_{\text {wall }}=20 \mathrm{~m} / \mathrm{s}$. 


\section{Thermal Transpiration Flow}

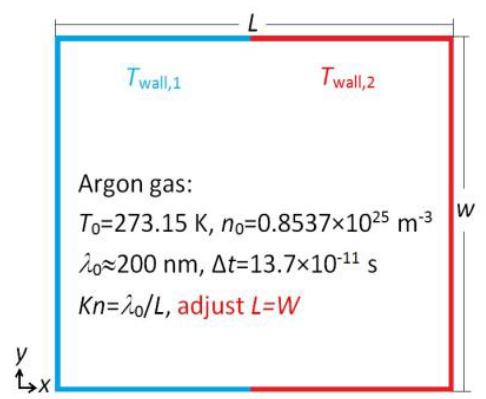

FIGURE 4. Schematic model of closed thermal transpiration flow.

This problem was studied first in [7] where $T_{\text {wall, }, 2} / T_{\text {wall, } 1}=2$. We set $T_{\text {wall, }, 2} / T_{\text {wall, } 1}=1.05 T_{0} / 0.95 T_{0} \approx 1.105$ to show the high efficiency of DSBGK simulations at low velocity. The Maxwell boundary condition is used and cell number is $40 \times 40$ for $K n=0.2$. Each cell contains about 1000 simulated molecules and $v=v(\kappa)$ in the DSBGK simulation. Fig. 5 shows the transient DSBGK results (no average) at $160^{\text {th }} \Delta t$ taking about 8 minutes of computational time, which implies that the computational time for each $\Delta t$ increased compared to that of Fig. 2 at $K n=0.063$. The same observation can be made when comparing the computational time used by Figs. 2 and 3. Main reason is that the frequency of molecular reflection on the wall increased with $K n$ and more computational time is used to generate random fractions. The transient $n$ and $T$ are smooth but the transient $u$ and $v$ contain large stochastic noises, which is because that the variation of $T$ is the active factor and has strong correlation with the variation of $n$ through the rough balance of pressure. However, the variations of $u$ and $v$ are passive factors and so sensitive to stochastic noise.

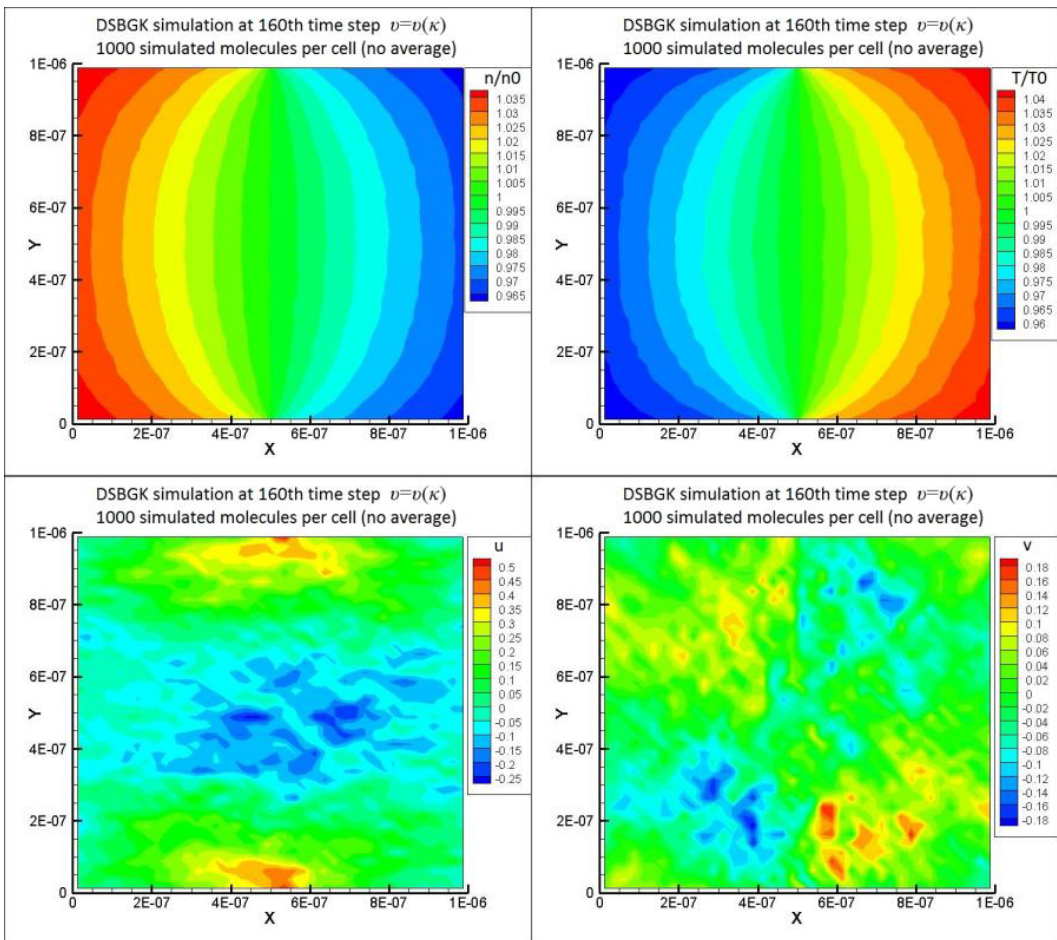

FIGURE 5. Transient DSBGK results at $160^{\text {th }} \Delta t, K n=0.2, T_{\text {wall, } 1}=0.95 T_{0}, T_{\text {wall }, 2}=1.05 T_{0}, 8$ minutes of CPU time.

In order to present smooth results of $u$ and $v$ for clear verification, we use the time-average process after $160 \Delta t$ to reduce noise and collect 1500 samples (sampling at each $\Delta t$ ) making the total computational time about 79 minutes. The DSBGK smooth results are given in Fig. 6 with comparison by the DSMC time-average results. As we can see, the DSBGK simulation using $v=v(\kappa)$ agrees very well with the DSMC simulation in predicting $n$ and $T$ and has small 
deviations in predicting $u$ and $v$ in the thermal transpiration problem. The DSBGK results using $v=v(\mu)$ are given together to show the dependence of $v$ on different problems. The comparison shows that we should select $v=v(\kappa)$ in thermal transpiration problem. In addition, Fig. 6 shows the agreement between the ensemble-average and time-average for sampling $u$ and $v$ in the DSBGK simulation, which is consistent with the conclusion of Fig. 3 that the time-average process is valid for sampling $u$ and $v$.

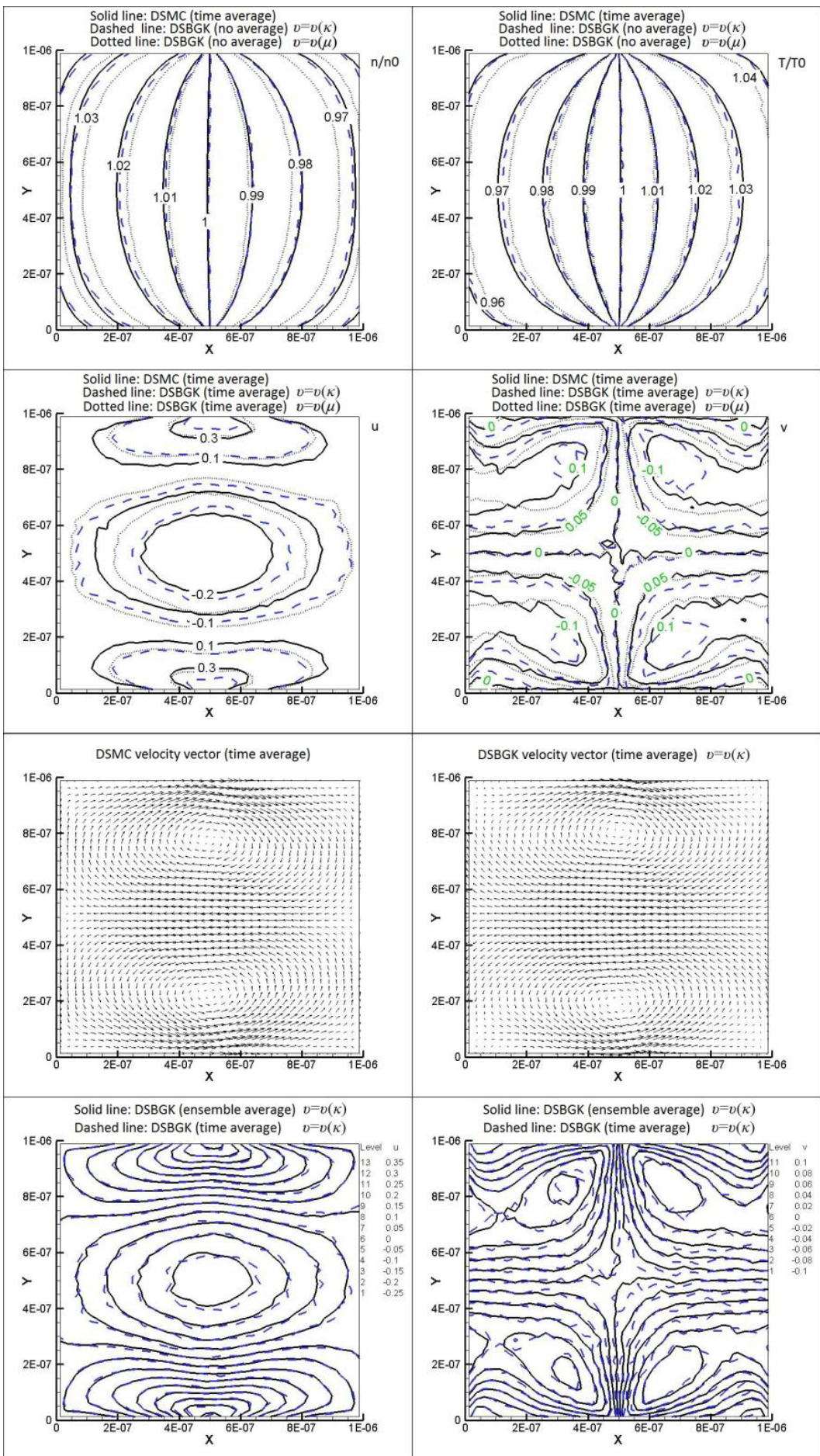

FIGURE 6. Comparison between DSMC and DSBGK methods, $K n=0.2, T_{\text {wall, } 1}=0.95 T_{0}, T_{\text {wall, }, 2}=1.05 T_{0}$. 


\section{Channel Flow}

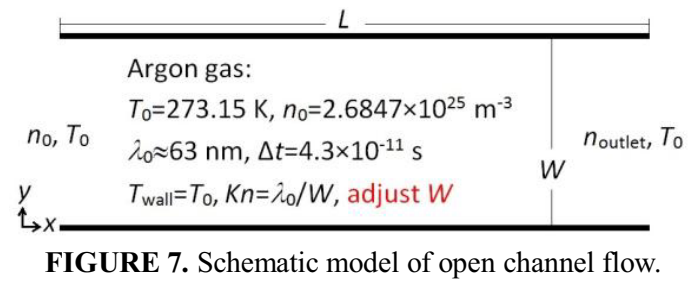

The DSBGK simulation of channel flows driven by pressure difference were reported first in [2] where $L=1$ micron. Here, $L=5$ microns and $W$ is regulated to change $K n$. The Maxwell boundary condition is used and cell number is $200 \times 20$ for $K n=0.63$. We set $v=v(\mu)$ and select $N_{l, t=0}$ appropriately such that each cell contains about 10 simulated molecules at the initial state to show the stability improvement of DSBGK simulations in open problems. The number densities are $n_{0}$ and $0.6 n_{0}$ at the inlet and outlet, respectively. In order to make the number of the simulated molecules per cell almost uniform, we choose $N_{l, \text { init,inlet }} / N_{l, \text { init,outlet }}=n_{\text {inlet }} / n_{\text {outlet }}=1 / 0.6$. Specifically, we set $N_{l, \text { init,inlet }}=N_{l, t=0} n_{\text {inlet }} / n_{0}=N_{l, t=0}$ and $N_{l, \text { init,outlet }}=N_{l, t=0} n_{\text {outlet }} / n_{0}=0.6 N_{l, t=0}$ to maintain the number of simulated molecules per cell approximately equal to 10 during the simulation process. After convergence, the transient DSBGK results at $30000^{\text {th }} \Delta t$ are given in Fig. 8 taking about 36 minutes of computational time. It shows that the DSBGK simulation is stable when using about 10 simulated molecules per cell in open problems. Using few simulated molecules reduces the memory usage and improves the applicability in problems of large scale. The transient $n$ is smooth but $T, u$, $v$ contain large stochastic noise. We use the time-average process to reduce noise and collect 6000 samples (sampling at each $\Delta t$ ) after $30000 \Delta t$, which takes about 8 minutes making the total computational time about 44 minutes. The DSBGK time-average results are given in Fig. 9 with comparison by the DSMC time-average results. Unfortunately, the average $v$ and $T$ of the DSBGK and DSMC simulations are still dominated by the stochastic noises due to small variations inside the flow domain, particularly in the area far away from the two ends. It should be pointed out that the average $v$ and $T$ can distinctly show their main variations near the inlet and outlet. Note that the dominance of stochastic noise is due to not only small characteristic velocity but also small variation. As we can see from Fig. 2 where $U_{\text {wall }}$ is only $0.1 \mathrm{~m} / \mathrm{s}$, the transient velocity distribution is smooth during the whole evolution process as its variation inside the whole flow domain is obvious. The agreement between the DSBGK time-average and transient results of $n$ implies that the unphysical fluctuation of $n$ is eliminated in the open problem. The magnitude of stochastic noise in the DSBGK time-average results using a small sample size is much smaller than that in the DSMC time-average results using a large sample size. Detailed comparisons with smoother $T / T_{\text {wall }}$ between the DSBGK and DSMC methods are made in [2] at $K n=0.63$ and 1.26 combined with different values of $\alpha_{\tau}$ and $\alpha_{\mathrm{n}}$.

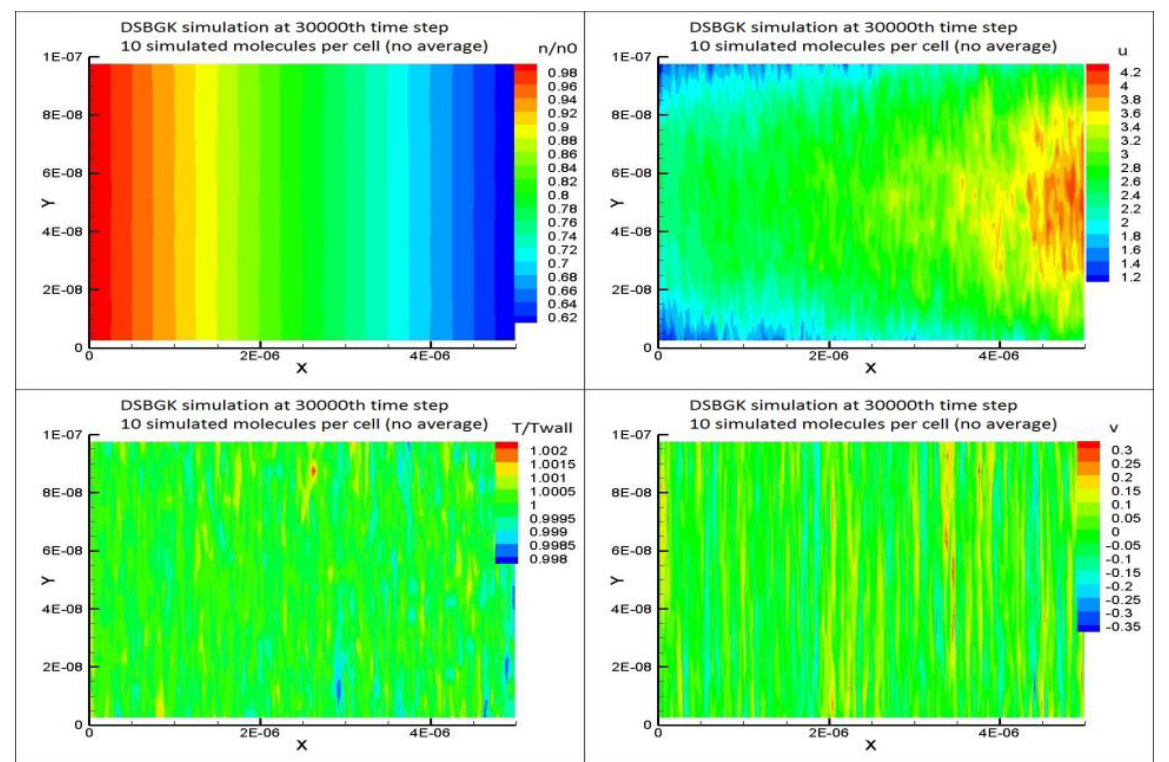

FIGURE 8. Transient DSBGK results at $30000^{\text {th }} \Delta t, K n=0.63, n_{\text {inlet }}=n_{0}, n_{\text {outlet }}=0.6 n_{0}, 36$ minutes of CPU time. 


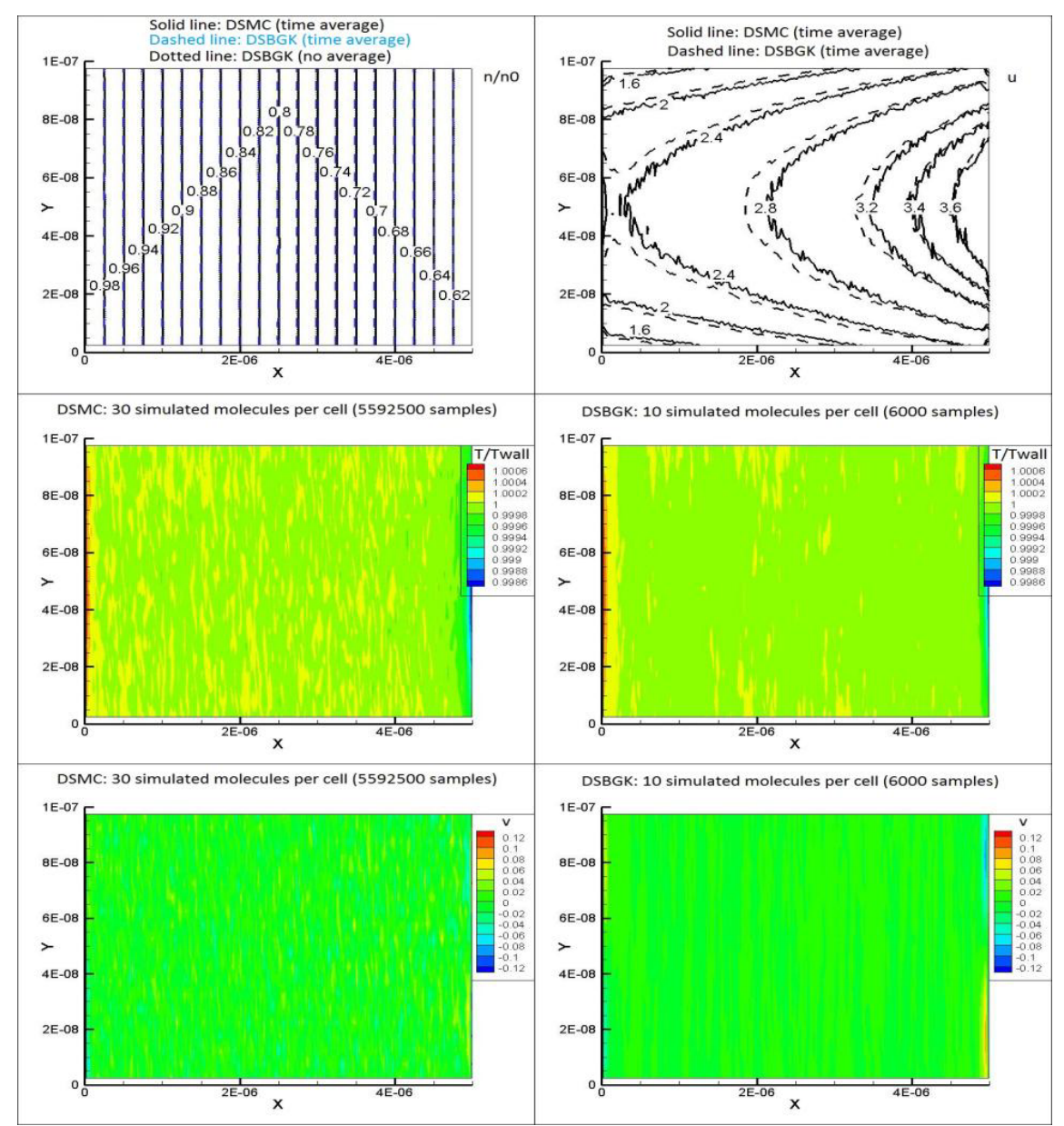

FIGURE 9. Comparison between DSMC and DSBGK methods, $K n=0.63, n_{\text {inlet }}=n_{0}, n_{\text {outlet }}=0.6 n_{0}$.

\section{CONCLUSIONS}

The DSBGK transient results are usually smooth but will be dominated by stochastic noise when the variations inside the flow domain are very small. In closed problems, the density distribution of DSBGK simulation is subject to unphysical fluctuation and the time-average process is invalid for sampling quantities related to density. But, the time-average process is still valid for sampling velocity and temperature. In open problems, the unphysical fluctuation is eliminated by the fixed density at the open boundary and the time-average process, if necessary, is always valid. The DSBGK simulation requires many simulated molecules in closed problems due to the unphysical fluctuation of density but is stable even when using about 10 simulated molecules per cell in open problems.

\section{REFERENCES}

1. Jun Li, Direct Simulation method based on BGK equation, 27th International Symposium on Rarefied Gas Dynamics, AIP Conference Proceedings 1333: 283-288, 2011.

2. Jun Li, Simulation of micro gas flows by the DS-BGK method, ASME 9th International Conference on Nanochannels, Microchannels and Minichannels, no. 58010, 2011.

3. Jun Li, Comparison between the DSMC and DSBGK methods, arXiv:1207.1040v1 [physics.comp-ph], 2012.

4. G. A. Bird, Molecular Gas Dynamics and the Direct Simulation of Gas Flows, Clarendon Press, Oxford, 1994.

5. C. Cercignani, M. Lampis, Transp. Theory Stat. Phys. 1, 101-114 (1971).

6. R. G. Lord, Phys. Fluids A 3, 706-710 (1991).

7. K. Aoki, S. Takata, H. Aikawa and F. Golse, Phys. Fluids 13, 2645-2661 (2001). 